\title{
Ein neues Chrommineral in Serbien
}

von

\author{
Milorad Z. Jovitschitsch.
}

Aus dem Laboratorium der Bergbau-Akademie in Belgrad.

(Vorgelegt in der Sitzung am 15. Oktober 1908.)

Unter Željin, einem Ausläufer des Gebirges Kopaonik, findet sich in größerer Ausbreitung ein mit den herabfließenden Bächen heruntergeschwemmter, kleinkörniger, glitzernder Sand, bestehend aus dunklen und hellen Partien, welchen noch der wahre Sand beigemengt ist. Durch einen Magneten lassen sich die dunklen, matten Partien, welche leicht von diesem herangezogen werden, von den hellen, glänzenden absondern, beiden aber, besonders den letzteren, haften noch immer große Mengen von Silikatverunreinigungen an, unter welchen deutlich $\mathrm{zu}$ erkennen sind Krystalle von Quarz, Liskun, Granat und andere weniger gut charakteristische mineralogische Individuen. Beide Teile stellen Krystalle, und zwar reguläre Oktaeder dar mit oft sehr deutlich ausgebildeten, regelmäßigen Flächen. Die Hauptmasse des Sandes repräsentieren glänzende Krystalle; von den matten sind nur etwas über $2 \%$ vorhanden.

Durch den Magneten grob voneinandergetrennt, sind sie bei weitem noch nicht für die Analysen geeignet; besonders die glänzenden Krystalle, welche äußerst mühsam von den sie massenhaft begleitenden Silikatbestandteilen mechanisch und nur unvollständig getrennt werden können. Ebensowenig lassen sie sich durch verschiedene, zu diesem Zwecke zu gebrauchenden Flüssigkeiten trennen. Auch đie matten Partien, die aus zermalmter Masse, leichter von Silikaten mittels des Magneten 
zu befreien sind, erwiesen sich als verschieden stark von diesem angezogen, weshalb auch sie weiter gereinigt werden mußten.

Unter einem vertikal befestigten Magneten wurden matte Oktaeder auf einem Papier ausgebreitet, auf die Entfernungen von 8 bis $5 \mathrm{~mm}$ diesem genähert und das dabei Angezogene in eine Fraktion Nr. I gesammelt. Dann wurden die Krystalle, welche auf die Entfernungen von 5 bis $2 \mathrm{~mm}$ angezogen waren, als Fraktion Nr. II bezeichnet, und schließlich diejenigen, welche nur bei Berührung vom Magneten angezogen werden, als Fraktion Nr. III angesehen. Indem diese Manipulation noch einige Male bei jeder Fraktion vorgenommen und jede für sich von noch etwaigen Verunreinigungen durch helle Krystalle und Silikatbestandteile befreit wurde, waren alle drei Fraktionen analysenbereit. Die kleinsten Mengen bekam man aus der Sammlung Nr. I; aus $100 \mathrm{~g}$ meistens nur gegen $0.2 \%$. Die beiden anderen sind in fast gleich großen Quantitäten vorhanden.

Bedeutend schwieriger aber lassen sich die anderen Oktaeder reinigen, da bei ihnen der ganze Rest von silikatartigen Beimengungen zurückbleibt. Und doch geschah es nach verschiedenen vergeblichen Versuchen am bequemsten durch Wasser auf folgende Weise.

In ein Becherglas, in welches der unmagnetische Rest des Sandes gebracht wird, gießt man soviel destilliertes Wasser, bis sein Niveau nur 2 bis $3 \mathrm{~cm}$ über die Sandschichte reicht, dann wird vorsichtig das Glas nur um soviel auf eine Seite geneigt, damit die obere Fläche dieser von Wasser berührt und nachher das Glas wieder langsam in vertikale Lage gebracht wird. Die Krystalle werden dabei nebst sehr geringen Mengen Silikaten auf die Wasseroberfläche fortgetragen und mittels eines Löffels auf ein Filtrierpapier gebracht. Man wiederholt diese Operation, sooft noch die Krystalle in Überschuß sich sammeln lassen (das Umrühren mit einem Glasstab bezweckt nichts, im Gegenteil wird dadurch das Hinaufstreben der Partikelchen gestört). Nach einiger Zeit bleibt der Sand zurück, derart verarmt an glänzenden Krystallen, daß sich ihre Ausscheidung nicht mehr lohnt. Getrocknet auf dem Filtrierpapier und bei $100^{\circ}$ lassen sie sich jetzt leicht von Verunreinigungen 
befieien: von Silikatbeimengungen mechanisch und die eventuellen magnetischen Beimengungen durch den Magneten. Auf diese Weise kann man in einer Stunde über $20 \mathrm{~g}$ analysenreines Mineral bekommen.

\section{Untersuchung der glänzenden Krystalle.}

Die Krystalle sind äußerst hart und lösen sich in keiner der Mineralsäuren auf, mögen sie noch so feingepulvert sein. Ebenso ist ihre Aufschließung durch Schmelzen mit Soda und Pottasche in Beisein von Kaliumnitrat und Kaliumchlorat oder Schwefel eine sehr schwierige und unvollständige. Dagegen wurden sie leicht aufgeschlossen durch Glühen mit Kalk in Anwesenheit von Alkalicarbonaten, und zwar am besten im Verhältnis: auf $1 g$ Substanz etwas mehr als $1 g$ von diesen (gleich große Mengen von Natrium- und Kaliumcarbonat) und $1.5 \mathrm{~g}$ Calciumoxyd.

Die in einem Platintiegel während einer Stunde lang über einem starken Gebläse geglühte Masse schmilzt nicht, sondern sintert nur zusammen und ist leicht vom Tiegel loszutrennen. Mit einem Glasstab zerkleinert und bei bedecktem Glase mit verdünnter Salzsäure behandelt, löst sie sich in dieser vollkommen klar auf. Um jedwede Reaktion, die zwischen Salzsäure und Chromat entstehen könnte, zu vermeiden, ist es vorteilhaft, die geglühte Masse mit warmem Wasser zu behandeln, den Rückstand von Ferrioxyd in Salzsäure aufzunehmen, beide Flüssigkeiten vereinigt mit Ammoniak zu neutralisieren und bis zur Trockne einzudampfen. Der so erhaltene Rückstand löst sich im Wasser, welchem ein paar Tropfen Salzsäure zugezetzt sind, vollständig klar auf, was bedeutet, daß die Krystalle keine Silikate sind, noch solche als Beimengungen enthalten. Nachdem aus der intensiv gelb gefärbten Lösung alles Eisen mittels Ammoniak gefällt und überschüssiges Ammon verdampft, wurde die abfiltrierte Lösung mit Salzsäure und Alkohol reduziert und mit Ammoniak alles Chrom ausgefällt. Beide Niederschläge waren durch Auswaschen mit heißem Wasser fast vollkommen von Alkali- und Erdalkalisalzen befreit. 
Hier die Analysen:

I. $0.304 \mathrm{~g}$ Substanz ergaben $0.1545 \mathrm{~g} \mathrm{Fe}_{2} \mathrm{O}_{3}$ und $0.1530 \mathrm{~g} \mathrm{Cr}_{2} \mathrm{O}_{3}$ oder $35 \cdot 52 \% \mathrm{Fe}$ und $34 \cdot 07 \% \mathrm{Cr}$.

II. $0.2783 g$ Substanz ergaben $0.1441 g \mathrm{Fe}_{2} \mathrm{O}_{3}$ und $0.1369 \mathrm{~g} \mathrm{Cr}_{2} \mathrm{O}_{3}$ oder $34 \cdot 68 \% \mathrm{Fe}$ und $33 \cdot 78 \% \mathrm{Cr}$.

III. $0.1775 g$ Substanz ergaben $0.0882 g \mathrm{Fe}_{2} \mathrm{O}_{3}$ und $0.0903 g \mathrm{Cr}_{2} \mathrm{O}_{3}$ oder $34 \cdot 85 \% \mathrm{Fe}$ und $33 \cdot 75 \% \mathrm{Cr}$.

Das geglühte Eisenoxyd, nebenbei gesagt, stark magnetisch, wurde mit primärem Kaliumsulfat geschmolzen, das erhaltene Ferrisalz mit Zink und Salzsäure reduziert und die Lösung mit Kaliumpermanganat in Beisein von $\mathrm{MnSO}_{4}$ titriert. Es erwies sich, daß alles fast reines Eisenoxyd war und nur unter $0.5 \%$ Aluminiumoxyd enthielt. Für $0.1 \mathrm{~g}$ Eisenoxyd wurden $7 \cdot 1 \mathrm{~cm}^{3}$ des Kaliumpermanganats verbraucht, dessen Koeffizient für Sauerstoff 0.000813 betrug.

Mit Natriumphosphat geprüft, erwies sich die von Eisen und Chrom befreite Lösung magnesiumhältig. Es waren über $2 \frac{1}{2} \% \mathrm{Mg}$ darin gefunden, aber als der von Kahlbaum seit Jahren bestellte und zur Aufschließung des Minerals verwendete Kalk auf Magnesium geprüft wurde, erwies er sich auch magnesiumhältig, so daß dessen wahre Menge im Mineral selbst mir unbekannt blieb; auf jeden Fall kann sie nicht viel über $1 \%$ ausmachen. Vorläufig verzichte ich auf seine genaue Bestimmung, worauf ich später, bis ich über die Genesis des Minerals auf dem Terrain selbst Studien gemacht habe, zurückkommen werde.

Aus den analytischen Daten springen sofort in die Augen die fast gleichgroßen Mengen von Eisen und Chrom, woraus man unbedingt den Schluß ziehen muß, daß die glänzenden Oktaeder nichts mit den Chromitoktaedern Gemeinsames haben. In dem theoretisch reinen Chromit, welcher als solcher niemals in der Natur vorkommt, steht die Menge des Eisens zu derjenigen des Chroms fast wie $1: 2$ oder $24 \cdot 88 \% \mathrm{Fe}: 46 \cdot 67 \% \mathrm{Cr}$. Auch in natürlich vorkommenden Chromiten bleibt das Eisen weit zurück hinter dem Chrom auf deren Kosten, und zwar des Eisens Magnesium, seltener zweiwertiges Chrom und noch seltener Calcium, und auf denen des Chroms Aluminium und 
dreiwertiges Eisen vorzukommen pflegen. In keiner der mir zu Gebote stehenden Chromitanalysen ist die Eisenmenge so hoch. ${ }^{1}$ Außerdem unterscheiden sich diese Krystalle von den Chromitkrystallen durch ihren hellen, metallischen Glanz, während letztere nur halbmetallischen Glanz besitzen. Schließlich ist das charakteristischeste Merkmal zur Unterscheidung von Mineralien, das spezifische Gewicht, ein verschiedenes. Chromeisenstein hat spezifisches Gewicht $4 \cdot 4$ bis $4 \cdot 6$, wogegen dieses Chrommineral nur gegen $3 \cdot 1$ hat. Somit ist sicher erwiesen, daß diese metallglänzenden, sehr harten Oktaeder einem neuen Chrommineral angehören.

Was seine Konstitution oder vielmehr chemische Formel anbelangt, so ist sie leicht zu ergründen. Wenn man von den geringen Mengen des Magnesiums und Aluminiums absieht, so resultiert aus den analytischen Daten glatt folgendes Atomverhältnis von Eisen, Chrom und Sauerstoff

$$
1: 1: 3
$$

woraus sich die Formel $\mathrm{FeCrO}_{3}$ oder $\mathrm{Fe}_{2} \mathrm{Cr}_{2} \mathrm{O}_{6}$ aufstellen läßt. Demnach wären diese Krystalle als eine Verbindung von Ferrioxyd mit Chromioxyd

$$
\mathrm{Fe}_{2} \mathrm{Cr}_{2} \mathrm{O}_{6}=\mathrm{Fe}_{2} \mathrm{O}_{3} \cdot \mathrm{Cr}_{2} \mathrm{O}_{3}
$$

aufzufassen, zum Unterschied von Chromit, welcher als eine Doppelverbindung von Ferrooxyd mit Chromoxyd angesehen wird. Oder noch einfacher als ein Chromioxyd, in welchem ein Chromatom durch ein Eisenatom, oder umgekehrt als ein Ferrioxyd, in welchem ein Eisenatom durch ein Chromatom vertreten ist. Das wäre ein wundervolles Beispiel für die Analogie dieser beiden Elemente.

\begin{tabular}{|c|c|c|c|c|}
\hline \multirow{2}{*}{\multicolumn{2}{|c|}{$\begin{array}{l}\text { Berechnet für } \\
\mathrm{FeCrO}_{3}\end{array}$}} & \multicolumn{3}{|c|}{ Gefunden } \\
\hline & & I & II & III \\
\hline & $35 \cdot 78$ & $35 \cdot 52$ & $34 \cdot 68$ & $34 \cdot 85$ \\
\hline & $33 \cdot 23$ & 34.07 & $33 \cdot 75$ & $33: 75$ \\
\hline & 30.99 & $30 \cdot 41$ & $31 \cdot 57$ & $31 \cdot 40$ \\
\hline
\end{tabular}

In 100 Teilen:

I Ramelsberg, Naumann-Zirkel Kenngott. 
Die gut übereinstimmenden Resultate beweisen nicht nur, daß man es mit einem mineralogischen Individuum, sondern $z u$ gleicher Zeit mit einem fast chemisch reinen Körper zu tun hat. $\mathrm{Da}$ er im Vergleich zu reinem Chromit weniger Chrom als dieser enthält, so schlage ich für dieses neue Chrommineral den Namen Chromitit vor.

Es ist vor einigen Jahren ein ähnlich zusammengesetztes Chromerz aus Kraubat in Obersteiermark beschrieben worden, ${ }^{1}$ dem man auf Grund seiner Analysen die Formel $\mathrm{FeCr}_{2} \mathrm{O}_{3} \mathrm{zu}-$ schrieb, so daß es also um ein Chromaton reicher wäre als der Chromitit. Indessen ist es weit davon entfernt, rein $z u$ sein; wie die Analysen zeigen:

$$
\begin{aligned}
& \mathrm{SiO}_{2} \ldots \ldots 4 \cdot 3 \\
& \begin{array}{lll}
\mathrm{MgO} & \ldots . . & 9 \cdot 7
\end{array} \\
& \mathrm{CaO} \ldots \ldots 6 \cdot 6 \\
& \mathrm{FeO} \ldots \ldots .9 \cdot 1 \\
& \mathrm{Al}_{2} \mathrm{O}_{3} \ldots \ldots 13 \cdot 7 \\
& \mathrm{Cr}_{2} \mathrm{O}_{3} \ldots \ldots 56 \cdot 2
\end{aligned}
$$

eher mit Chromit in Einklang zu bringen.

Über Chromitit, welcher mir seitens des Obersten Miloševitch, des Besitzers des Terrains, überbracht worden war, gedenke ich mit Hilfe einiger Fachgenossen Terrainstudien $z u$ machen, wo auch andere von gewöhnlichen Chromeisensteinen verschiedene Chromerze vorzukommen scheinen.

\section{Untersuchung der matten Krystalle.}

Dem Äußern, wie auch der Eigenschaft nach, vom Magneten angezogen zu werden, scheinen diese Krystalle dem Magnetit anzugehören. ${ }^{2}$ Wenn man sie aber analysiert, so zeigen sie noch ein bedeutendes Quantum an Chrom auf, dessen Ursprung der ganz und gar unmagnetische Chromitit unmöglich sein kann.

1 Zeitschrift für praktische Geologie für 1900, J. S. 339.

2 In der Zeitschrift für praktische Geologie für 1908 (Separatabdruck) erschien eine kurze Notiz aus dem Leobener geologisch-mineralogischen Institut, in welcher Chromitit als Chromit und diese matten Oktaeder als Magnetit bezeichnet wurden. 
Eine jede der drei eingangs erwähnten Fraktionen wurde für sich analysiert, woraus sich ergab, daß die erste, auch die kleinste, fast reiner Magnetit ist.

$0.1515 \mathrm{~g}$ ergaben $0.145 \mathrm{~g} \mathrm{Fe}_{2} \mathrm{O}_{3}$ und $0.0132 \mathrm{~g} \mathrm{Cr}_{2} \mathrm{O}_{3}$ oder $66.99 \% \mathrm{Fe}$ und $6.01 \% \mathrm{Cr}$.

Die Fraktionen mit Nr. II bezeichnet, welche aus verschiedenen Quantitäten des ursprünglichen Sandes gewonnen wurden, lieferten ungefähr die gleichen analytischen Resultate. Die Werte für Eisen und Chrom variierten zwischen folgenden Zahlen:

43 bis $45 \% \mathrm{Fe}$ und $21 \cdot 5$ bis $25 \% \mathrm{Cr}$.

und diejenigen des Magnesiums und Aluminiums waren sehr gering. Daraus kann man nur schließen, daß auch die matten Oktaeder einem Chrommineral zuzuschreiben sind. Der Gedanke war nahe, sie als eine Mischung aus Magnetit und Chromitit anzusehen, womit der höhere Wert an Eisen und der geringere an Chrom in Einklang stünde. Dieser Deutung widersetzt sich aber die Tatsache, daß Chromitit absolut unmagnetisch ist. Andrerseits kann auch das Magnetit in erheblicheren Mengen nicht zugegen sein, da es, wie bekannt, durch längeres gelindes Erwärmen mit Salzsäure von dieser gelöst wird, was ich nicht habe nachweisen können. Aus denselben Gründen fällt auch der Zweifel fort, daß Magnetit mit Chromit vermengt sein kann. Weil sie sich aber neben dem Chromitit als Hauptmasse jenes Sandes befinden, so weist dieser Umstand auf die Möglichkeit hin, daß sie in naher Beziehung zu Chromitit stehen können. Und da sie von Magneten angezogen werden im Gegensatz zu Chromitit, zweifelsohne infolge ihres größeren Reichtums an Eisen, so faßte ich sie als eine Doppelverbindung aus zwei Molekülen Ferrioxyd mit einem Molekül Chromioxyd

$$
2 \mathrm{Fe}_{2} \mathrm{O}_{3} \cdot \mathrm{Cr}_{2} \mathrm{O}_{3}
$$

auf. Die Theorie verlangt für einen solchen chemisch reinen Körper

$$
47 \cdot 35 \% \mathrm{Fe} \text { und } 22 \cdot 19 \% \mathrm{Cr} \text {. }
$$

Diese Auffassung wird noch durch den Umstand unterstützt, daß die Krystalle der Fraktion Nr. III aus verschiedenen 
Quantitäten des Sandes erhalten, sich mit ihren Werten an Eisen und Chrom denjenigen des Chromitits nähern. So z. B. schwankt ihr Eisenwert $z$ wischen 38 bis $34 \%$ und derjenige des Chroms zwischen 36 bis $34 \%$. Sie werden sehr schwer, oft nur durch direkte Berührung mit dem Magneten von diesem angezogen, welche Eigenschaft mit dem verminderten Quantum an Eisen in Einklang steht. Andrerseits kann man unter ihren matten Krystallen auch viele solche mit metallischem, wenn auch nicht so wie beim Chromitit ausgeprägtem Glanze beobachten. Aus all dem kann man schließen, daß diese Krystalle ein Übergangsstadium zu den Chromititkrystallen bilden. Meine weitere Aufgabe wird sein, auf dem Terrain selbst die Muttersubstanz dieser Chrommineralien aufzusuchen. 